\title{
A New Approach to Modification of Rearrangements in Metallorganic Chemistry of Phosphorus, Arsenic, Antimony and Bismuth
}

\author{
Aibassov Yerkin Zhakenovich*, Yemelyanova Valentina Stepanovna, Shakieva Tatyana Vladimirovna, Aibassov \\ Gizatulla, Abenov Bakhyt and Bulenbayev Maksat \\ Research Institute of New Chemical Technologies and Materials, Kazakh National University Al-Farabi, Almaty 005012, Kazakhstan
}

\begin{abstract}
We discovered a new approach modification Bamberger, Barton, Beckmann, Wallach, Gabriel, Hofmann, Hofmann A.W. Martius, Dimroth, Semmler-Wolff-Schroeter, Sus, Claisen, Newman-Kwart, Orton, Pistschimuka, Robev, Smiles, Sawdey, Sommelet, Stevens, Tiemann, Fischer-Hepp, Chapman, Chattaway, Schonberg, Stieglitz Rearrangements with of phosphorous, arsine, stibine and bismuthine in organometallic chemistry. The authors have proposed a new mechanism for possible reactions.
\end{abstract}

Key words: Rearrangements, organic compounds of arsenic, antimony, bismuth.

\section{Introduction}

Have now been discovered a lot of new reactions of organometallic chemistry of phosphorus, arsenic, antimony and bismuth. The resulting novel compounds have potential biologically active substances and can be used in medicine.

We were interested to analyze the classical rearrangement in organic chemistry and try to modify them, and to expand the scope of their application.

In this paper the 26 classical rearrangements in order to study the possibility of expanding and modifying them by replacing the nitrogen atoms to phosphorus, arsenic, antimony and bismuth.

\section{Theory}

It is known that Bamberger Rearrangement arilnitramines in o-nitroanilines by acids $\left(\mathrm{H}_{2} \mathrm{SO}_{4}\right.$, $\mathrm{CH}_{3} \mathrm{COOH}$, $\left.\mathrm{HCOOH}\right)[1,2]$ :

$\mathrm{R}-\mathrm{C}_{6} \mathrm{H}_{4}-\mathrm{NR}^{\prime}-\mathrm{NO}_{2} \rightarrow \mathrm{R}-\mathrm{C}_{6} \mathrm{H}_{4}-\mathrm{N}+\mathrm{HR}^{\prime}-\mathrm{NO}_{2} \rightarrow$

$\left[\mathrm{R}-\mathrm{C}_{6} \mathrm{H}_{4}-\mathrm{N}^{+} \mathrm{HR}-\mathrm{O}-\mathrm{NO} \rightarrow \mathrm{R}-\mathrm{C}_{6} \mathrm{H}_{3}=\mathrm{N}^{+} \mathrm{HR}^{\prime}\left(\mathrm{NO}_{2}\right)\right] \rightarrow$

\footnotetext{
*Corresponding author: Aibassov Yerkin Zhakenovich, Professor, research field: metalorganic chemistry of uranium, As, Sb and Bi. E-mail: erkin53@mail.ru.
}

$$
\mathrm{R}\left(\mathrm{NO}_{2}\right) \mathrm{C}_{6} \mathrm{H}_{3}-\mathrm{NHR}^{\prime}
$$

where, $\mathrm{R}=\mathrm{H}, \mathrm{CH}_{3}, \mathrm{NO}_{2}, \mathrm{Cl}, \mathrm{Br}$; $\mathrm{R}^{\prime}=\mathrm{H}, \mathrm{CH}_{3}$.

It is known that Bamberger arylhydroxilamins in $\mathrm{n}$-aminophenols in the presence of mineral acids $\left(\mathrm{H}_{2} \mathrm{SO}_{4}\right)$ [3]:

$$
\begin{gathered}
\mathrm{R}-\mathrm{C}_{6} \mathrm{H}_{4}-\mathrm{NHOH} \rightarrow\left[\mathrm{R}^{-} \mathrm{C}_{6} \mathrm{H}_{4}-\mathrm{NHO}+\mathrm{H}_{2}+\mathrm{H}_{2} \mathrm{O} \rightarrow\right. \\
\left.\mathrm{R}(\mathrm{HO}) \mathrm{C}_{6} \mathrm{H}_{3}=\mathrm{NH}\right] \rightarrow \mathrm{R}(\mathrm{HO}) \mathrm{C}_{6} \mathrm{H}_{4}-\mathrm{NH}_{2}
\end{gathered}
$$

It is known that Barton photochemical rearrangement of nitrites in nitroso compounds by intramolecular exchange nitroso on hydrogen bonded to $\gamma$-carbon atom, followed by isomerization to oximes, or dimerization $[4,5]$ :

$$
\begin{gathered}
\mathrm{NO}_{2}-\mathrm{C}_{4} \mathrm{H}_{8}-\mathrm{CH}(\mathrm{H})-\mathrm{C}_{3} \mathrm{H}_{6} \mathrm{CH}_{3}+\mathrm{h} v \rightarrow \\
\mathrm{O}_{-} \mathrm{C}_{4} \mathrm{H}_{8}-\mathrm{CH}(\mathrm{H})-\mathrm{C}_{3} \mathrm{H}_{6} \mathrm{CH}_{3}+* \mathrm{NO} \rightarrow \\
\mathrm{HO}-\mathrm{C}_{4} \mathrm{H}_{8}-\mathrm{C} * \mathrm{H}-\mathrm{C}_{3} \mathrm{H}_{6} \mathrm{CH}_{3}+* \mathrm{NO} \rightarrow \\
\mathrm{HO}-\mathrm{C}_{4} \mathrm{H}_{8}-\mathrm{CH}(\mathrm{NO})-\mathrm{C}_{3} \mathrm{H}_{6} \mathrm{CH}_{3} \rightarrow \text { Dimer }+ \\
\mathrm{HO}-\mathrm{C}_{4} \mathrm{H}_{8}-\mathrm{C}(=\mathrm{NOH})-\mathrm{C}_{3} \mathrm{H}_{6} \mathrm{CH}_{3}
\end{gathered}
$$

Reacting a aliphatic, aliphatic-aromatic and alicyclic compounds and compounds containing various functional groups (Hal, $\mathrm{OR}$ and et.):

$$
\begin{gathered}
\mathrm{C}_{6} \mathrm{H}_{5}-\left(\mathrm{CH}_{2}\right)_{4} \mathrm{ONO}+\mathrm{h} v \rightarrow \\
{\left[\mathrm{C}_{6} \mathrm{H}_{5}-\mathrm{CH}(\mathrm{NO})-\left(\mathrm{CH}_{2}\right)_{2} \mathrm{CH}_{2} \mathrm{OH}\right]_{2}} \\
\left(\mathrm{CH}_{2}\right)_{3} \mathrm{CHONO}+\mathrm{h} v \rightarrow \mathrm{ONCH}_{2}\left(\mathrm{CH}_{2}\right)_{2} \mathrm{CHO}
\end{gathered}
$$


The reaction is used in steroid chemistry, as they allow to introduce functional groups in the molecule inactive space.

It is known that Beckmann Rearrangement An acid-induced rearrangement of oximes to give amides [6-9]:<smiles>[R]NC([R])=O</smiles>

This reaction is related to the Hofmann and Schmidt Reactions and the Curtius Rearrangement, in that an electropositive nitrogen is formed that initiates an alkyl migration. Mechanism of the Beckmann
Rearrangement following:<smiles>ON=C1CCCCC1</smiles><smiles></smiles>

Oximes generally have a high barrier to inversion, and accordingly this reaction is envisioned to proceed by protonation of the oxime hydroxyl, followed by migration of the alkyl substituent "trans" to nitrogen. The N-O bond is simultaneously cleaved with the expulsion of water, so that formation of a free nitrene is avoided.<smiles>[TeH]C1[NH+]=C2CCCCC21</smiles>

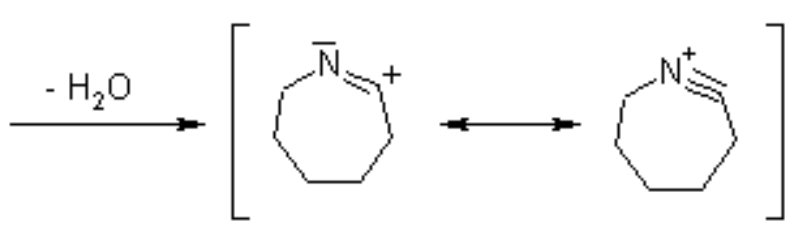
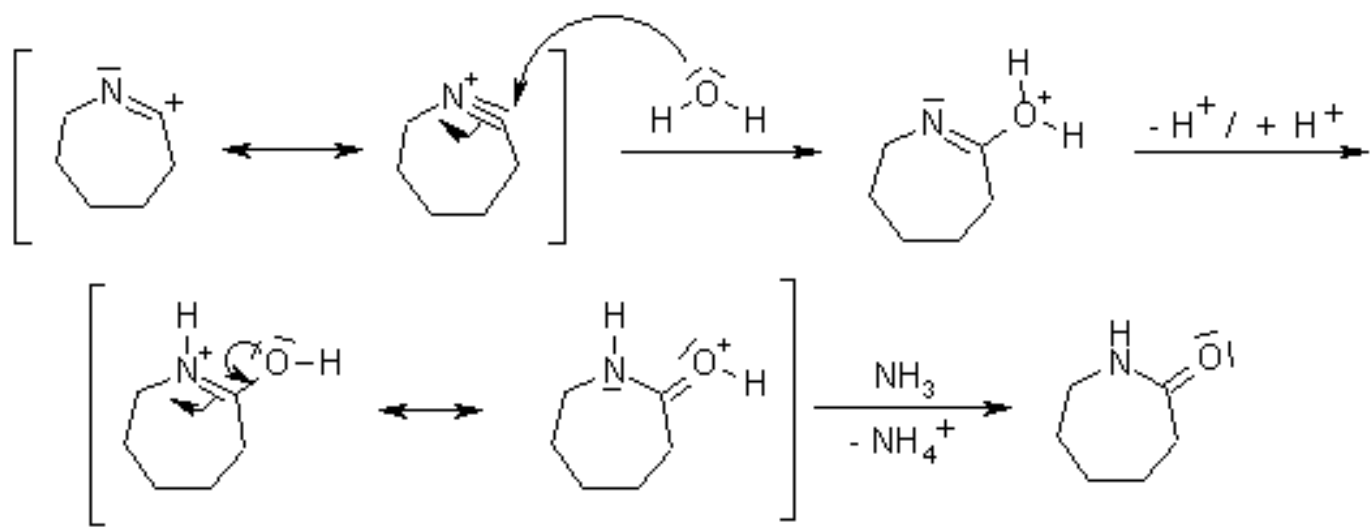

It is known that Wallach rearrangement azoxybenzenes in p-oksiazobenzols when heated by the action of sulfuric acid [10-15]:

$\mathrm{H}-\mathrm{C}_{6} \mathrm{H}_{4}-\mathrm{N}=\mathrm{N}(\mathrm{O})-\mathrm{C}_{6} \mathrm{H}_{5} \rightarrow \mathrm{HO}-\mathrm{C}_{6} \mathrm{H}_{4}-\mathrm{N}=\mathrm{N}-\mathrm{C}_{6} \mathrm{H}_{5}(9)$

Electron-donating substituents, and the substituents cause steric hindrance, prevent reaction.

Known that Gabriel rearrangement $\mathrm{N}$-carboxymethyl- and N-phenaculphtalimids in a substituted 1,4-dioxiizohinolins under the influence of sodium alcoholate RONa [16-19]:

$$
\begin{gathered}
\mathrm{C}_{6} \mathrm{H}_{4}(\mathrm{CO})_{2} \mathrm{NCH}_{2} \mathrm{COR} \rightarrow \\
\mathrm{C}_{6} \mathrm{H}_{4}(\mathrm{COOR})-\mathrm{CONHCH}{ }_{2} \mathrm{COR} \rightarrow \\
\mathrm{C}_{9} \mathrm{H}_{4} \mathrm{~N}(\mathrm{OH})(\mathrm{OH}) \mathrm{COR}
\end{gathered}
$$

where, $\mathrm{R}=\mathrm{OR}$, $\mathrm{n}_{-} \mathrm{C}_{6} \mathrm{H}_{4} \mathrm{R}^{\prime \prime}\left(\mathrm{R} "=\mathrm{H}, \mathrm{CH}_{3}, \mathrm{OCH}_{3}, \mathrm{Cl}\right.$, $\left.\mathrm{Br}, \mathrm{NO}_{2}\right) ; \mathrm{R}^{\prime}=\mathrm{CH}_{3}, \mathrm{C}_{2} \mathrm{H}_{5}$.

Similarly (N-izathinyl) acetic ester derivative rearranges to 3,4-dioksihinoline:

$$
\begin{gathered}
\mathrm{C}_{6} \mathrm{H}_{4} \mathrm{C}(=\mathrm{O}) \mathrm{C}(=\mathrm{O}) \mathrm{NCH}_{2} \mathrm{COOC}_{2} \mathrm{H}_{5} \rightarrow \\
\mathrm{C}_{9} \mathrm{H}_{4} \mathrm{~N}(\mathrm{OH})(\mathrm{OH}) \mathrm{COOC}_{2} \mathrm{H}_{5}
\end{gathered}
$$

It is believed that during the reaction there is an opening of the imide ring and rearrangement imide anion in a carbanion, followed by intramolecular Dieckmann condensation.

The starting compounds are prepared by alkylation of phthalimide potassium halocarboxylic acids or esters phenylacylhalogenids. 
It is known that the Hofmann rearrangement is the organic reaction of a primary amide to a primary amine with one fewer carbon atom [20-25].

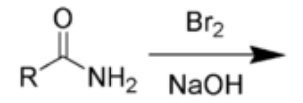

$$
\begin{aligned}
& {\left[\mathrm{R}_{\mathrm{N}}=\mathrm{C}=\mathrm{O}\right] \underset{-\mathrm{CO}_{2}}{\stackrel{\mathrm{H}_{2} \mathrm{O}}{\longrightarrow}} \mathrm{R}-\mathrm{NH}_{2}}
\end{aligned}
$$

The reaction of bromine with sodium hydroxide forms sodium hypobromite in situ, which transforms the primary amide into an intermediate isocyanate via a formation of a nitrene. The intermediate isocyanate is hydrolyzed to a primary amine, giving off carbon dioxide.

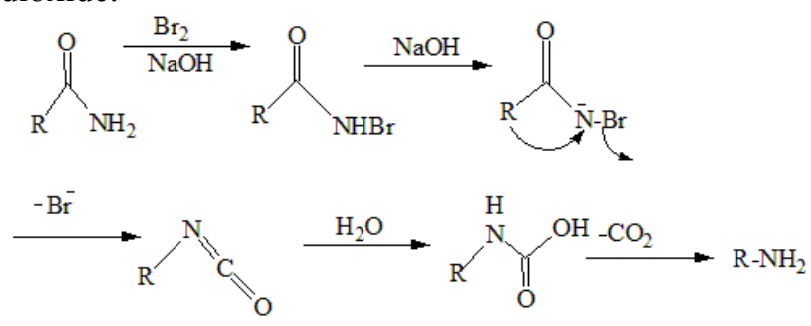

Several reagents can substitute for bromine. Lead tetraacetate,

$\mathrm{N}$-bromosuccinimide, (bis(trifluoroacetoxy)iodo) benzene, and DBU (1,8-diazabicyclo[5.4.0]undec-7-ene) can effect a Hofmann rearrangement. In the following example, the intermediate isocyanate is trapped by methanol, forming a carbamate.

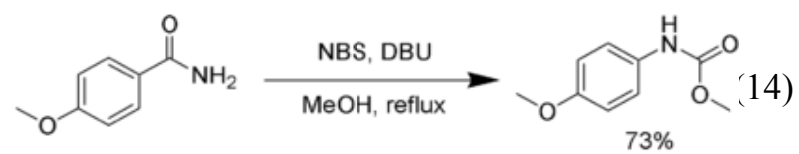

In a similar fashion, the intermediate isocyanate can be trapped by tert-butyl alcohol, yielding the tert-butoxycarbonyl (Boc)-protected amine.

Known that the Hofmann A.W. Martius Thermal rearrangement of $\mathrm{N}$-alkylanilines hydrochlorides in C-alkylanilines [26-29]:

$$
\begin{gathered}
\mathrm{C}_{6} \mathrm{H}_{5} \mathrm{NHR} \cdot \mathrm{HX} \rightarrow\left[\mathrm{C}_{6} \mathrm{H}_{5} \mathrm{NH}_{2}+\mathrm{R}^{+}+\mathrm{X}^{-}\right] \rightarrow \\
\mathrm{R}-\mathrm{C}_{6} \mathrm{H}_{4} \mathrm{NH}_{2} \cdot \mathrm{HX}
\end{gathered}
$$

where, $\mathrm{X}=\mathrm{Cl}$, Br, J.

This reaction also come $\mathrm{N}, \mathrm{N}$-dialkylanilines. Mainly formed of para-isomers. The migration of primary alkyl groups occurs at a higher temperature than the secondary. If $\mathrm{R}$ is a branched chain, in the process of the rearrangement is also isomerization radical, e.g.:

$$
\begin{gathered}
\mathrm{C}_{6} \mathrm{H}_{5} \mathrm{NH}\left(\mathrm{CH}_{2}\right)_{2} \mathrm{CH}\left(\mathrm{CH}_{3}\right)_{2} \rightarrow \\
\mathrm{n}-\left(\mathrm{CH}_{3}\right)_{3} \mathrm{CCH}_{2}-\mathrm{C}_{6} \mathrm{H}_{4} \mathrm{NH}_{2}
\end{gathered}
$$

Modification Reilly-Hickinbottom-rearrangement in the presence of metal halides $\left(\mathrm{ZnCl}_{2}, \mathrm{CdCl}_{2}\right)$ :

$$
\mathrm{C}_{6} \mathrm{H}_{5} \mathrm{NHR} \cdot \mathrm{HX} \rightarrow \mathrm{R}-\mathrm{C}_{6} \mathrm{H}_{4} \mathrm{NH}_{2} \cdot \mathrm{HX}
$$

Known that the Dimroth Isomerization of 1-substituted-1,2-dihydro-2-iminopirimidinov under the action of bases in a 2-substituted aminopyrimidines (amidine rearrangement) [30]:

$$
\mathrm{R}^{\prime}-\mathrm{C}_{4} \mathrm{H}_{2} \mathrm{NR}=\mathrm{NH} \rightarrow \mathrm{R}^{\prime}-\mathrm{C}_{4} \mathrm{H}_{2} \mathrm{~N}_{2} \text {-NHR }
$$

where, $\mathrm{R}=\mathrm{Alk}, \mathrm{CH}_{2} \mathrm{COOC}_{2} \mathrm{H}_{5} ; \mathrm{R}^{\prime}=\mathrm{Alk}, \mathrm{Hal}, \mathrm{NO}_{2}$, $\left(\mathrm{CH}_{3}\right)_{2} \mathrm{~N}, \mathrm{CONH}_{2}$.

Electron-withdrawing groups on the pyrimidine ring accelerate rearrangement. The reaction rate increases in the number of substituents $\mathrm{R}$ (the highest rate of reaction at the methyl radical):

$$
\begin{gathered}
\mathrm{CH}_{3}<\mathrm{C}_{2} \mathrm{H}_{5}<\mathrm{C}_{4} \mathrm{H}_{9}<\mathrm{C}_{7} \mathrm{H}_{15}<\mathrm{CH}_{2} \mathrm{CH}=\mathrm{CH}_{2}< \\
\mathrm{CH}_{2} \mathrm{C}_{6} \mathrm{H}_{5}<\mathrm{CH}_{2} \mathrm{C}_{6} \mathrm{H}_{4} \mathrm{NO}_{2} \text {-n. }
\end{gathered}
$$

In non-aqueous media rearrangement slows down. Rearrangement is general and is observed in a number of heterocyclic systems, especially for triazines, tetrazoles, thiadiazoles, adenines.

It is known that the Semmler-Wolff-Schroeter Rearrangement of oximes $\alpha, \beta$-Unsaturated alicyclic ketones in aromatic amines under the action of a mixture of the Beckmann $\left(\left(\mathrm{CH}_{3} \mathrm{CO}\right)_{2}+\mathrm{CH}_{3} \mathrm{COOH}\right.$, $\left.\mathrm{HCl}, 100{ }^{\circ} \mathrm{C}\right)$ [31-40]:

$$
\begin{gathered}
\mathrm{R}-\mathrm{C} 6 \mathrm{H} 8=\mathrm{NOH}+\left(\mathrm{CH}_{3} \mathrm{CO}\right)_{2}+\mathrm{CH}_{3} \mathrm{COOH} \rightarrow \\
{\left[\mathrm{R}-\mathrm{C}_{6} \mathrm{H}_{8}=\mathrm{NOCOCH}_{3} \rightarrow\right.} \\
\left.\mathrm{R}-\mathrm{C}_{6} \mathrm{H}_{8}=\mathrm{N}+\mathrm{HOCOCH}_{3}\right] \rightarrow \\
\mathrm{R}-\mathrm{C}_{6} \mathrm{H}_{4}-\mathrm{NH}_{2}
\end{gathered}
$$

where, $\mathrm{R}=\mathrm{H}$, Alk, pyridyl.

This reaction takes disubstituted ketones oximes and ketones outside the fused system ( $\alpha$-tetralone, 1-ketotetragidrofenantren).

Known that the Sus photochemical rearrangement of o-hinondiazidov in ketenes, accompanied by contraction cycle [41-44]: 


$$
\begin{gathered}
\mathrm{C}_{10} \mathrm{H}_{6}(=\mathrm{O})=\mathrm{N}^{+}=\mathrm{N}^{-} \leftrightarrow \mathrm{C}_{10} \mathrm{H}_{6}\left(\mathrm{O}^{-}\right)-\mathrm{N}^{+}=\mathrm{N}+\mathrm{h} \nu \rightarrow \\
{\left[\mathrm{C}_{10} \mathrm{H}_{6}^{+}\left(\mathrm{O}^{-}\right) \leftrightarrow \mathrm{C}_{10} \mathrm{H}_{6}^{+}\left(\mathrm{O}^{-}\right)\right] \rightarrow \mathrm{C}_{9} \mathrm{H}_{6}=\mathrm{C}=\mathrm{O}+\mathrm{H}_{2} \mathrm{O} \rightarrow} \\
\mathrm{C}_{9} \mathrm{H}_{6}-\mathrm{COOH}
\end{gathered}
$$

The reaction is a convenient method of obtaining a number of acids cyclopentadiene.

Regrouping undergo various cyclic $\alpha$-diazo ketones (alicyclic, heterocyclic compounds of terpene series).

The starting o-hinondiazidy diazotization receive appropriate o-aminohinons.

Known that the Claisen Thermal rearrangement of allyl ethers of phenols or enols in the isomeric C-allyl derivatives [45-52]:

$$
\begin{array}{r}
-\mathrm{CH}=\mathrm{CH}-\mathrm{O}-\mathrm{CH}_{2} \mathrm{CH}=\mathrm{CHR} \rightarrow \\
{\left[\mathrm{O}=\mathrm{CHCH}_{2}-\mathrm{CHR}-\mathrm{CH}=\mathrm{CH}_{2}\right] \rightarrow} \\
\mathrm{HO}-\mathrm{CH}=\mathrm{CH}-\mathrm{CH}(\mathrm{R}) \mathrm{CH}=\mathrm{CH}_{2}
\end{array}
$$

where, $\mathrm{R}=\mathrm{H}$, Alk. $\mathrm{C}_{6} \mathrm{H}_{5}$.

Examples of reactions:

$$
\begin{aligned}
& \mathrm{R}-\mathrm{C}_{6} \mathrm{H}_{4}-\mathrm{O}-\mathrm{CH}_{2} \mathrm{CH}=\mathrm{CHR}^{\prime} \rightarrow \\
& \mathrm{R}-\mathrm{C}_{6} \mathrm{H}_{3}(\mathrm{OH}) \mathrm{CHR}^{\prime} \mathrm{CH}=\mathrm{CH}_{2} \\
& \mathrm{CH}_{2}=\mathrm{CH}-\mathrm{O}-\mathrm{CH}_{2} \mathrm{CH}=\mathrm{CH}_{2} \rightarrow \\
& \mathrm{CH}_{2}=\mathrm{CHCH}_{2}-\mathrm{CH}_{2} \mathrm{CHO}
\end{aligned}
$$

Similar rearrangement observed in heterocycles such:

$$
\begin{aligned}
\mathrm{C}_{6} \mathrm{H}_{4} \mathrm{ZN}=\mathrm{C}-\mathrm{OCH}_{2} \mathrm{CH}=\mathrm{CH}_{2} \rightarrow \\
\mathrm{C}_{6} \mathrm{H}_{4} \mathrm{ZN}\left(-\mathrm{CH}_{2}-\mathrm{CH}=\mathrm{CH}_{2}\right) \mathrm{C}=\mathrm{O}
\end{aligned}
$$

where, $\mathrm{Z}=\mathrm{O}$, $\mathrm{S}$.

This reaction is widely used in the synthesis of natural compounds, such as essential oils.

Like allyl ether enamines rearrange to form imines (amino-Claisen rearrangement - amino-Claisen):

$$
\begin{aligned}
\mathrm{CH}_{2} & =\mathrm{CH}-\mathrm{CH}_{2}-\mathrm{NR}-\mathrm{CH}=\mathrm{C}(\mathrm{R})-\mathrm{CH}_{3} \rightarrow \\
\mathrm{CH}_{2} & =\mathrm{CH}-\mathrm{CH}_{2}-\mathrm{C}(\mathrm{R})\left(\mathrm{CH}_{3}\right)-\mathrm{CH}=\mathrm{NR}
\end{aligned}
$$

where, $\mathrm{R}=\mathrm{Alk}, \mathrm{C}_{6} \mathrm{H}_{5}$.

Undergo a similar rearrangement unsaturated thioesters (thio-Claisen rearrangement - thio-Claisen):

$$
\begin{gathered}
\mathrm{CH}_{2}=\mathrm{CH}-\mathrm{S}-\mathrm{CH}_{2}-\mathrm{CH}=\mathrm{CH}_{2} \rightarrow \\
\mathrm{CH}_{2}=\mathrm{CH}-\mathrm{CH}_{2}-\mathrm{CH}_{2}-\mathrm{CH}=\mathrm{S} \\
\mathrm{CH}_{2}=\mathrm{CH}-\mathrm{S}-\mathrm{CH}_{2}-\mathrm{CH}=\mathrm{CH} \rightarrow \\
\mathrm{CH}_{2}=\mathrm{C}=\mathrm{CH}-\mathrm{CH}_{2}-\mathrm{CH}=\mathrm{S} \rightarrow \mathrm{C}_{5} \mathrm{H}_{7} \mathrm{~S}
\end{gathered}
$$

It is known that the Newman-Kwart Thermal rearrangement of O-aryltiocarbamats in S-aryltiocarbanats [53-55]:

$$
\operatorname{Ar}-\mathrm{O}-\mathrm{C}(=\mathrm{S})-\mathrm{NR}_{2} \rightarrow \operatorname{Ar}-\mathrm{S}-\mathrm{C}(=\mathrm{O})-\mathrm{NR}_{2}
$$

Alkaline hydrolysis of S-aryltiocarbamats leads to the corresponding thiols.

The reaction is used as one of the steps of recovery of phenols, which is not suitable for the direct hydrogenation:

$$
\begin{gathered}
\mathrm{Ar}-\mathrm{OH}+\mathrm{COS}+\mathrm{NH}\left(\mathrm{C}_{2} \mathrm{H}_{5}\right)_{2} \\
\mathrm{Ar}-\mathrm{O}-\mathrm{CSN}\left(\mathrm{C}_{2} \mathrm{H}_{5}\right)_{2} \rightarrow \\
\mathrm{Ar}-\mathrm{S}-\mathrm{CON}\left(\mathrm{C}_{2} \mathrm{H}_{5}\right)_{2}+\mathrm{KOH} \rightarrow \\
\mathrm{Ar}-\mathrm{SH}+\mathrm{H}_{2} \rightarrow \mathrm{ArH}
\end{gathered}
$$

It is known that the Orton Rearrangement of $\mathrm{N}$-halogeno-N-atsilarilaminov in o- and n-galogenarilamidy by acids $\left(\mathrm{CH}_{3} \mathrm{COOH}\right)$ [56-58]:

$$
\begin{gathered}
\text { R- } \mathrm{C}_{6} \mathrm{H}_{4}-\mathrm{N}(\mathrm{X}) \mathrm{COR}+\mathrm{HX} \rightarrow \\
{\left[2 \mathrm{R}-\mathrm{C}_{6} \mathrm{H}_{4}-\mathrm{NHCOR}+\mathrm{X}_{2}\right] \rightarrow} \\
\text { R- } \mathrm{C}_{6} \mathrm{H}_{3}(\mathrm{NHCOR} \prime)(\Pi-\mathrm{X})+ \\
\text { R- } \mathrm{C}_{6} \mathrm{H}_{3}(\mathrm{NHCOR})(\mathrm{o}-\mathrm{X})
\end{gathered}
$$

In the resulting mixture prevails para-isomer. Regrouping and goes for naphthalene derivatives.

Regrouping can take place in non-aqueous media by the action of $\mathrm{ClCH}_{2} \mathrm{COOH}, \mathrm{C}_{6} \mathrm{H}_{5} \mathrm{CH}_{2} \mathrm{COOH}$, o- or $\mathrm{m}-\mathrm{O}_{2} \mathrm{NC}_{6} \mathrm{H}_{4} \mathrm{COOH}$ :

$$
\begin{gathered}
\mathrm{C}_{6} \mathrm{H}_{5} \mathrm{NXCOR}+\mathrm{R}^{\prime} \mathrm{COOH} \rightarrow \\
{\left[\mathrm{C}_{6} \mathrm{H}_{5} \mathrm{NHCOR}+\mathrm{R}^{\prime} \mathrm{COOX}\right] \rightarrow} \\
\mathrm{X}-\mathrm{C}_{6} \mathrm{H}_{5} \mathrm{NHCOR}+\mathrm{R}^{\prime} \mathrm{COOH}
\end{gathered}
$$

Known that the Pistschimuka the conversion of aromatic amines to azo compounds by the action of selenium (or sulfur) and merkuratsetamide [59, 60]:

$$
\begin{gathered}
2 \mathrm{ArNH}_{2}+\mathrm{Se}+\left(\mathrm{CH}_{3} \mathrm{CONH}\right)_{2} \mathrm{Hg} \rightarrow \\
\mathrm{Ar}-\mathrm{N}=\mathrm{N}-\mathrm{Ar}
\end{gathered}
$$

where, $\mathrm{Ar}=\mathrm{C}_{6} \mathrm{H}_{4} \mathrm{R}, \mathrm{R}=\mathrm{H}, \mathrm{CH}_{3}, \mathrm{OCH}_{3}, \mathrm{NO}_{2}, \mathrm{Cl}$, $\mathrm{N}\left(\mathrm{CH}_{3}\right)_{2}$.

Merkuracetamide serves to bind the liberated hydrogen selenide (or hydrogen sulphide). Instead merkuratsetamide can be used mercury salts with inorganic or organic acids.

It is known that Robev rearrangement phenylhydrazones aromatic aldehydes by reacting a N-phenylamidines with alkali metal amides $\left(\mathrm{KNH}_{2}\right.$, $\mathrm{NaNH}_{2}$ ) in the presence of oxidants by heating [61, 62]: 


$$
\begin{gathered}
\mathrm{ArCH}=\mathrm{N}-\mathrm{NHC}_{6} \mathrm{H}_{5}+\mathrm{NaNH}_{2}+\mathrm{O}_{2} \rightarrow\left[\mathrm{ArCH}=\mathrm{N}^{\cdot}\right. \\
\left.+\mathrm{NHC}_{6} \mathrm{H}_{5} \rightarrow \mathrm{ArC}=\mathrm{NH}+\mathrm{NHC}_{6} \mathrm{H}_{5}\right] \rightarrow \\
\operatorname{ArC}(=\mathrm{NH})-\mathrm{NHC}_{6} \mathrm{H}_{5} \quad(80 \%)
\end{gathered}
$$

Reacting a phenylhydrazones also certain heterocyclic aldehydes.

Known that the Smiles Isomerization of substituted aromatics (diaryl ethers diarilsulfids, sulfoxids, sulfonyl, aryl esters of carboxylic acids and sulphonic aromatic) under the action of alkalis resulting intramolecular nucleophilic substitution [63-66]:

$$
\begin{aligned}
& \mathrm{R}-\mathrm{C}_{6} \mathrm{H}_{3}(\mathrm{ZH}) \mathrm{Y}-\mathrm{C}_{6} \mathrm{H}_{4}-\mathrm{R}^{\prime} \rightarrow \\
& {\left[\mathrm{R}-\mathrm{C}_{6} \mathrm{H}_{3}(\mathrm{Z}-)-\mathrm{Y}^{-} \mathrm{C}_{6} \mathrm{H}_{4}-\mathrm{R}^{\prime} \rightarrow\right.} \\
& \left.\mathrm{R}-\mathrm{C}_{6} \mathrm{H}_{3}(\mathrm{Y}-)-\mathrm{Z}-\mathrm{C}_{6} \mathrm{H}_{4}-\mathrm{R}^{\prime}\right] \\
& \mathrm{R}-\mathrm{C}_{6} \mathrm{H}_{3}(\mathrm{YH})-\mathrm{Z}-\mathrm{C}_{6} \mathrm{H}_{4}-\mathrm{R}^{\prime}
\end{aligned}
$$

where, $\mathrm{R}=\mathrm{H}, \mathrm{CH}_{3}, \mathrm{Hal}$; R' $=\mathrm{H}, \mathrm{NO}, \mathrm{NO}_{2}, \mathrm{Hal}$; $\mathrm{Y}=\mathrm{S}$, $\mathrm{SO}, \mathrm{SO}_{2}, \mathrm{SO}_{2} \mathrm{O}, \mathrm{O}, \mathrm{COO} ; \mathrm{ZH}=\mathrm{OH}, \mathrm{SH}, \mathrm{NHOH}, \mathrm{NH}_{2}$, $\mathrm{SO}_{2} \mathrm{H}, \mathrm{OR} ", \mathrm{CONHR} ", \mathrm{SO}_{2} \mathrm{NHR}^{\prime}, \mathrm{NHR} "$ (where R" = Alk, Ar).

The reaction is facilitated by the ability to increase the nucleophilic anion of $\mathrm{Z}$ - and in the presence of electron-acceptor R'.

Similarly rearranged compound containing both one aromatic condensed system radicals, heterocyclic, or aliphatic radical:

$$
\begin{gathered}
\mathrm{Cl}-\mathrm{C}_{5} \mathrm{H}_{2} \mathrm{~N}\left(\mathrm{NHCOCH}_{3}\right)-\mathrm{S}_{-} \mathrm{C}_{6} \mathrm{H}_{4} \mathrm{NO}_{2} \rightarrow \\
\mathrm{Cl}-\mathrm{C}_{5} \mathrm{H}_{2} \mathrm{~N}\left(\mathrm{SCH}_{3}\right)-\mathrm{N}\left(\mathrm{COCH}_{3}\right)-\mathrm{C}_{6} \mathrm{H}_{4} \mathrm{NO}_{2} \\
\mathrm{C}_{6} \mathrm{H}_{4}\left(\mathrm{NO}_{2}\right)-\mathrm{SO}-\mathrm{CH}_{2} \mathrm{CH}_{2} \mathrm{OH} \rightarrow \\
\mathrm{C}_{6} \mathrm{H}_{4}\left(\mathrm{NO}_{2}\right)-\mathrm{O}-\mathrm{CH}_{2} \mathrm{CH}_{2} \mathrm{SOH}
\end{gathered}
$$

If the nucleophilicity of the anion $\mathrm{Y}^{-}$and $\mathrm{Z}^{-}$is close, the rearrangement may be reversible, e.g.:

$$
\begin{gathered}
\mathrm{H}_{3} \mathrm{C}-\mathrm{C}_{6} \mathrm{H}_{3}\left(\mathrm{SO}_{2} \mathrm{H}\right)-\mathrm{O}-\mathrm{C}_{6} \mathrm{H}_{4} \mathrm{NO}_{2} \leftrightarrow \\
\mathrm{H}_{3} \mathrm{C}-\mathrm{C}_{6} \mathrm{H}_{3}(\mathrm{OH})-\mathrm{SO}_{2}-\mathrm{C}_{6} \mathrm{H}_{4} \mathrm{NO}_{2}
\end{gathered}
$$

Reaction Truce-Smiles rearrangement of o-metildiarilsulfons under the $\mathrm{n}-\mathrm{C}_{4} \mathrm{H}_{9} \mathrm{Li}$ :

$\mathrm{R}-\mathrm{C}_{6} \mathrm{H}_{3}\left(\mathrm{CH}_{3}\right)-\mathrm{SO}_{2} \mathrm{Ar} \rightarrow \mathrm{R}-\mathrm{C}_{6} \mathrm{H}_{3}\left(\mathrm{SO}_{2} \mathrm{H}\right)-\mathrm{CH}_{2} \mathrm{Ar}(38)$

Unlike Smiles rearrangement in this case easily migrate unactivated aromatic residue, in particular naphthyl.

Known that the Sawdey Rearrangement 4-arilazooksazolidonov-5-substituted in 1,2,4-triazole under the presence of bases $(\mathrm{KOH})$ [67-69]:

$$
\begin{aligned}
& \operatorname{ArNHN}=\mathrm{C}_{3} \mathrm{NO}(=\mathrm{O})-\mathrm{C}_{6} \mathrm{H}_{5} \\
& \text { HOOC- } \mathrm{C}_{3} \mathrm{~N}_{3}(\mathrm{Ar})-\mathrm{C}_{6} \mathrm{H}_{5}
\end{aligned}
$$

where, $\mathrm{Ar}=\mathrm{C}_{6} \mathrm{H}_{5}, \mathrm{C}_{6} \mathrm{H}_{4} \mathrm{CH}_{3}, \mathrm{C}_{6} \mathrm{H}_{4} \mathrm{OCH}_{3}, \mathrm{C}_{6} \mathrm{H}_{4} \mathrm{Br}$.

It is known that the Sommelet Regrouping in trialkylbenzylammonium salts in tertiary amines containing o-alkylbenzyl group, under the action of strong bases [70-72]:

$$
\begin{gathered}
\mathrm{C}_{6} \mathrm{H}_{5}-\mathrm{CH}(\mathrm{R})-\mathrm{N}^{+} \mathrm{R}_{2}{ }^{\prime}-\mathrm{CH}_{2} \mathrm{R}^{\prime \prime} \rightarrow \\
{\left[\mathrm{CHR}=\mathrm{C}_{6} \mathrm{H}_{4}-\mathrm{CH}\left(\mathrm{R}^{\prime \prime}\right)-\mathrm{NR}_{3}{ }^{\prime}\right]} \\
\mathrm{CH}_{2} \mathrm{R}-\mathrm{C}_{6} \mathrm{H}_{4}-\mathrm{CH}\left(\mathrm{R}^{\prime \prime}\right)-\mathrm{NR}_{2}{ }^{\prime}
\end{gathered}
$$

It is known that the Stevens intramolecular rearrangement of quaternary ammonium salts of tertiary amines in the presence of bases [73]:

$$
\begin{gathered}
\mathrm{RCH}_{2}-\mathrm{N}^{+}\left(\mathrm{CH}_{2} \mathrm{Ar}\right)\left(\mathrm{CH}_{3}\right)_{2} \mathrm{Br}^{-} \rightarrow \\
\mathrm{RCH}\left(\mathrm{CH}_{2} \mathrm{Ar}\right)-\mathrm{N}\left(\mathrm{CH}_{3}\right)_{2}
\end{gathered}
$$

Ability $\mathrm{ArCH}_{2}$-group migration depends on the nature of the substituent in the aryl ring, and increased in the series from $\mathrm{NO}_{2}$ to $\mathrm{CH}_{3} \mathrm{O}$ :

$$
\mathrm{CH}_{3} \mathrm{O}<\mathrm{H}, \mathrm{CH}_{3}<\mathrm{Cl}, \mathrm{Br}, \mathrm{J}<\mathrm{NO}_{2} \text {. }
$$

Undergo a similar rearrangement of salt sulfonium arsonium, stibonium, phosphonium.

The transformation in the series aminimids similar rearrangement Stevens rearrangement called Wawzonek [74-78]:

$$
\begin{gathered}
\mathrm{CH}_{3}-\mathrm{CO}-\mathrm{N}-\mathrm{N}^{+} \mathrm{R}\left(\mathrm{CH}_{3}\right)_{2} \rightarrow \\
\mathrm{CH}_{3}-\mathrm{CO}-\mathrm{NR}-\mathrm{N}\left(\mathrm{CH}_{3}\right)_{2}
\end{gathered}
$$

where, $\mathrm{R}=\mathrm{C}_{6} \mathrm{H}_{5} \mathrm{CH}_{3}, \mathrm{CH}_{2}-\mathrm{CH}=\mathrm{CH}_{2}$.

In the case of $\mathrm{R}^{\prime}=\mathrm{Alk}$ aminimid decomposed to isocyanate and a tertiary amine:

$$
\begin{gathered}
\mathrm{RCO}-\mathrm{N}-\mathrm{N}^{+} \mathrm{R}^{\prime}\left(\mathrm{CH}_{3}\right)_{2} \rightarrow \\
\mathrm{RN}=\mathrm{C}=\mathrm{O}+\left(\mathrm{CH}_{3}\right)_{2} \mathrm{NR}^{\prime}
\end{gathered}
$$

It is known that the Tiemann Rearrangement amidoximes carboxylic acid derivatives of urea by reaction with benzenesulfonyl chloride $\left(\mathrm{C}_{6} \mathrm{H}_{5} \mathrm{SO}_{2} \mathrm{Cl}\right)$ and subsequent hydrolysis $[79,80]$ :

$$
\begin{gathered}
\mathrm{R} \text { 'R"N-CR=NOH }+\mathrm{C}_{6} \mathrm{H}_{5} \mathrm{SO}_{2} \mathrm{Cl} \rightarrow \\
\mathrm{R}^{\prime} \mathrm{R} " \mathrm{~N}-\mathrm{CR}=\mathrm{N}-\mathrm{OSO}_{2} \mathrm{C}_{6} \mathrm{H}_{5} \rightarrow \mathrm{R}^{\prime} \mathrm{R} " \mathrm{~N}-\mathrm{CO}-\mathrm{NHR}
\end{gathered}
$$
where, $\mathrm{R}=\mathrm{C}_{6} \mathrm{H}_{5}, \mathrm{CH}_{2} \mathrm{C}_{6} \mathrm{H}_{5}$.

Speed rearrangement depends on the inductive effect of the substituents $\mathrm{R}^{\prime}$ and $\mathrm{R}^{\prime \prime}$ decreases in the order from $\mathrm{CH}_{3}$ to $\mathrm{H}$ : 
$\mathrm{R}=\mathrm{R}^{\prime}=\mathrm{CH}_{3}>\mathrm{R}=\mathrm{CH}_{3}, \mathrm{R}^{\prime}=\mathrm{C}_{6} \mathrm{H}_{5}>\mathrm{R}=\mathrm{R}^{\prime}=\mathrm{H}$.

When the cyclic rearrangement occurs amidoximes expansion cycle:

$$
\mathrm{C}_{6} \mathrm{H}_{11} \mathrm{~N}=\mathrm{N}-\mathrm{OSO}_{2} \mathrm{C}_{6} \mathrm{H}_{4} \mathrm{CH}_{3} \rightarrow \mathrm{C}_{6} \mathrm{H}_{11} \mathrm{~N}_{2} \mathrm{O}
$$

Amidoximes can easily be prepared from nitriles by the action of $\mathrm{NH}_{2} \mathrm{OH}$.

Known that the Fischer-Hepp Rearrangement of aromatic N-nitrosamines in n-nitrosoaniline under the influence of acids [81-84]:

$$
\begin{aligned}
& \mathrm{R}-\mathrm{C}_{6} \mathrm{H}_{4}-\mathrm{N}\left(\mathrm{R}^{\prime}\right)-\mathrm{NO} \rightarrow\left[\mathrm{R}^{-} \mathrm{C}_{6} \mathrm{H}_{4}-\mathrm{N}+\mathrm{H}\left(\mathrm{R}^{\prime}\right)-\mathrm{NO} \leftrightarrow\right. \\
& \left.\mathrm{R}-\mathrm{C}_{6} \mathrm{H}_{4}-\mathrm{NHR}+\mathrm{NO}^{+}\right] \rightarrow \mathrm{R}^{-} \mathrm{C}_{6} \mathrm{H}_{3}\left(\mathrm{NHR}^{\prime}\right)(\mathrm{NO})(46)
\end{aligned}
$$
where, $\mathrm{R}=$ Alk, $\mathrm{Hal}, \mathrm{COOH} ; \mathrm{R}^{\prime}=\mathrm{Alk}$, Ar.

Regrouping also exposed naphthalene derivatives. For example, $\mathrm{N}$-nitroso-N-alkyl- $\beta$-naphthylamines are converted into $\alpha$-nitroso-N-alkyl- $\beta$-naphthylamines.

Known that the Chapman Thermal isomerization of aromatic iminoether in $\mathrm{N}, \mathrm{N}$-diarilamidy aromatic carboxylic acids [85-87]:

$$
\operatorname{Ar}-\mathrm{C}(\mathrm{OAr} ")=\mathrm{N}-\mathrm{Ar} \mathrm{r}^{\prime} \rightarrow \mathrm{Ar}-\mathrm{CO}-\mathrm{N}\left(\mathrm{Ar}^{\prime \prime}\right)-\mathrm{Ar}^{\prime}
$$

where, $\mathrm{Ar}, \mathrm{Ar}^{\prime}=\mathrm{C}_{6} \mathrm{H}_{5}, \mathrm{C}_{6} \mathrm{H}_{4} \mathrm{Cl}, \mathrm{C}_{6} \mathrm{H}_{4} \mathrm{NO}_{2}, \mathrm{C}_{6} \mathrm{H}_{4} \mathrm{OCH}_{3}$, $\mathrm{C}_{6} \mathrm{H}_{4} \mathrm{CH}_{3} ; \mathrm{Ar}^{\prime \prime}=\mathrm{C}_{6} \mathrm{H}_{4} \mathrm{R}$ (where $\mathrm{R}=\mathrm{H}, \mathrm{F}, \mathrm{Cl}, \mathrm{Br}, \mathrm{NO}_{2}$, Alk), naphtyl.

Electron accept substituents in the radical Ar" facilitate and electron-donor substituents impede reaction.

It is known that the Chattaway Rearrangement of N, $\mathrm{N}$-diatsilanilins in $\mathrm{N}$-acetylaminoarilketons in the presence of $\mathrm{ZnCl}_{2}$ [88-94]:

$$
\mathrm{RC}_{6} \mathrm{H}_{4}-\mathrm{N}(\mathrm{COR})_{2} \rightarrow \mathrm{RC}_{6} \mathrm{H}_{3}(4-\mathrm{COR})(\mathrm{NHCOR})(48)
$$
where, $\mathrm{R}=\mathrm{Alk}, \mathrm{C}_{6} \mathrm{H}_{5} ; \mathrm{R}^{\prime}=\mathrm{H}, \mathrm{Cl}, \mathrm{Br}, \mathrm{CH}_{3}$.

Known that the Schonberg Thermal rearrangement diaryltioncarbonats in diaryltiolcarbonats [95-100]:

$$
\begin{gathered}
\mathrm{ArO}-\mathrm{C}(=\mathrm{S})-\mathrm{OAr} \rightarrow[\mathrm{Ar} \ldots \mathrm{S} \ldots \mathrm{S} \ldots \mathrm{C}-\mathrm{OAr}] \rightarrow \\
\operatorname{Ar}-\mathrm{S}-\mathrm{C}(=\mathrm{O})-\mathrm{OAr}
\end{gathered}
$$

Electron-donating substituents at the ortho or para-position to the oxygen atom markedly facilitate the reaction.

The reaction used to prepare and recover thiophenols and phenols:

$$
\begin{aligned}
2 \mathrm{ArOH}+\mathrm{SOCl}_{2} & \rightarrow \mathrm{ArO}-\mathrm{C}(=\mathrm{S})-\mathrm{OAr} \rightarrow \\
\mathrm{ArS}-\mathrm{CO}-\mathrm{OAr}+\mathrm{HO}^{-} & \rightarrow \mathrm{ArSH}+\mathrm{H}_{2} \rightarrow \mathrm{ArH}
\end{aligned}
$$

Known that the Stieglitz Rearranging triarylmethylhydroxylamines in Anils under the $\mathrm{PCl}_{5}$ :

$$
\begin{gathered}
\mathrm{ArAr}_{2}{ }^{\prime} \mathrm{C}-\mathrm{NHOH} \rightarrow \mathrm{Ar}_{2}{ }^{\prime} \mathrm{C}=\mathrm{N}-\mathrm{Ar}+ \\
\mathrm{ArAr}{ }^{\prime} \mathrm{C}=\mathrm{N}-\mathrm{Ar}^{\prime}
\end{gathered}
$$

The presence of electron-donor substituents in the aromatic ring facilitates the rearrangement. The ability of the aryl moiety to migrate decreases in the series from $\left(\mathrm{CH}_{3}\right)_{2} \mathrm{NC}_{6} \mathrm{H}_{4}$ to $\mathrm{O}_{2} \mathrm{NC}_{6} \mathrm{H}_{4}:\left(\mathrm{CH}_{3}\right)_{2} \mathrm{NC}_{6} \mathrm{H}_{4}>$ $\mathrm{CH}_{3} \mathrm{OC}_{6} \mathrm{H}_{4}>\mathrm{CH}_{3} \mathrm{C}_{6} \mathrm{H}_{4}>\mathrm{C}_{6} \mathrm{H}_{5}>\mathrm{O}_{2} \mathrm{NC}_{6} \mathrm{H}_{4}$.

Thus, the analysis of classical rearrangements showed that the nitrogen atom may be replaced by atoms of phosphorus, arsenic, antimony and bismuth. This opens up new possibilities for the synthesis of new organometallic compounds of phosphorus, arsenic, antimony and bismuth.

\section{Results and Discussion}

We propose to modify the Bamberger rearrangement arylnitr (phosphine, arsine, stibine, bismuthine) amine in o-nitro (P, As, $\mathrm{Sb}, \mathrm{Bi}$ ) anilines with acids $\left(\mathrm{H}_{2} \mathrm{SO}_{4}, \mathrm{CH}_{3} \mathrm{COOH}, \mathrm{HCOOH}\right)$ :

$$
\begin{gathered}
\mathrm{R}-\mathrm{C}_{6} \mathrm{H}_{5}-\mathrm{ER}^{\prime}-\mathrm{NO}_{2} \rightarrow\left[\mathrm{R}-\mathrm{C}_{6} \mathrm{H}_{5}-\mathrm{E}+\mathrm{HR}^{\prime}-\mathrm{NO}_{2} \rightarrow\right. \\
\left.\mathrm{R}-\mathrm{C}_{6} \mathrm{H}_{5} \text {-E }+\mathrm{HR} \text {-O-NO } \rightarrow \text { R-C } \mathrm{C}_{6} \mathrm{H}_{4}=\mathrm{E}+\mathrm{HR}^{\prime}\left(\mathrm{NO}_{2}\right)\right] \rightarrow \\
\mathrm{R}\left(\mathrm{NO}_{2}\right) \mathrm{C}_{6} \mathrm{H}_{4}-\mathrm{EHR}
\end{gathered}
$$

where, $\mathrm{E}=\mathrm{N}, \mathrm{P}, \mathrm{As}, \mathrm{Sb}, \mathrm{Bi}$; $\mathrm{R}=\mathrm{H}, \mathrm{CH}_{3}, \mathrm{NO}_{2}, \mathrm{Cl}, \mathrm{Br}$; $\mathrm{R}^{\prime}=\mathrm{H}, \mathrm{CH}_{3}$.

We propose to modify the Bamberger rearrangement arylhydroxyl (phosphine, arsine, stibine, bismuthine) amines in $n$ - (phosphine, arsine, stibine, bismuthine) aminophenols in the presence of mineral acids $\left(\mathrm{H}_{2} \mathrm{SO}_{4}\right)$ :

$$
\begin{gathered}
\mathrm{R}-\mathrm{C}_{6} \mathrm{H}_{4}-\mathrm{NHOH} \rightarrow\left[\mathrm{R}_{-}-\mathrm{C}_{6} \mathrm{H}_{4}-\mathrm{NHO}^{+} \mathrm{H}_{2}+\mathrm{H}_{2} \mathrm{O} \rightarrow\right. \\
\left.\mathrm{R}(\mathrm{HO}) \mathrm{C}_{6} \mathrm{H}_{2}=\mathrm{NH}\right] \rightarrow \mathrm{R}(\mathrm{HO}) \mathrm{C}_{6} \mathrm{H}_{3} \mathrm{NH}_{2}
\end{gathered}
$$

where, $\mathrm{E}=\mathrm{N}, \mathrm{P}, \mathrm{As}, \mathrm{Sb}, \mathrm{Bi}$.

We propose to modify the Barton rearrangement photochemical rearrangement nitr (arsen-, antimon-, bismuth) in a nitroso ( $\mathrm{P}, \mathrm{As}, \mathrm{Sb}, \mathrm{Bi})$ compounds by intramolecular exchange nitrozo(phosphorinozo, arsinozo, stibinazo, bismuthazo) groups on hydrogen bonded to $\gamma$-carbon atom followed by isomerization in ox $(\mathrm{P}, \mathrm{As}, \mathrm{Sb}, \mathrm{Bi})$ or dimerization: 


$$
\begin{gathered}
\mathrm{EO}_{2}-\mathrm{C}_{4} \mathrm{H}_{8}-\mathrm{CH}(\mathrm{H})-\mathrm{C}_{3} \mathrm{H}_{6} \mathrm{CH}_{3}+\mathrm{h} v \rightarrow \\
{\left[\mathrm{O}^{*}-\mathrm{C}_{4} \mathrm{H}_{8}-\mathrm{CH}(\mathrm{H})-\mathrm{C}_{3} \mathrm{H}_{6} \mathrm{CH}_{3}+* \mathrm{EO} \rightarrow\right.} \\
\left.\mathrm{HO}-\mathrm{C}_{4} \mathrm{H}_{8}-\mathrm{C} * \mathrm{H}-\mathrm{C}_{3} \mathrm{H}_{6} \mathrm{CH}_{3}+* \mathrm{EO}\right] \rightarrow \\
\mathrm{HO}-\mathrm{C}_{4} \mathrm{H}_{8}-\mathrm{CH}(\mathrm{EO})-\mathrm{C}_{3} \mathrm{H}_{6} \mathrm{CH}_{3} \rightarrow \text { Dimer }+ \\
\quad \mathrm{HO}-\mathrm{C}_{4} \mathrm{H}_{8}-\mathrm{C}(=\mathrm{EOH})-\mathrm{C}_{3} \mathrm{H}_{6} \mathrm{CH}_{3}
\end{gathered}
$$

where, $\mathrm{E}=\mathrm{N}, \mathrm{P}, \mathrm{As}, \mathrm{Sb}, \mathrm{Bi}$.

We propose to modify the Beckmann Rearrangement by replacing nitrogen atoms per atom of phosphorus, arsenic, antimony and bismuth:

$$
\mathrm{RR}^{\prime} \mathrm{C}=\mathrm{E}-\mathrm{OH} \rightarrow \mathrm{R}^{\prime} \mathrm{CO}-\mathrm{EH}-\mathrm{R}
$$

where, $\mathrm{E}=\mathrm{N}, \mathrm{P}, \mathrm{As}, \mathrm{Sb}, \mathrm{Bi}$.

We propose to modify the Wallach rearrangement azoxy (P, As, Sb, Bi) benzene p-oxiazo (P, As, Sb, Bi) benzene by heating under the action of sulfuric acid:

$$
\begin{gathered}
\mathrm{H}-\mathrm{C}_{6} \mathrm{H}_{4}-\mathrm{E}_{1}=\mathrm{E}_{2}(\mathrm{O})-\mathrm{C}_{6} \mathrm{H}_{5} \rightarrow \\
\mathrm{HO}-\mathrm{C}_{6} \mathrm{H}_{4}-\mathrm{E}_{1}=\mathrm{E}_{2}-\mathrm{C}_{6} \mathrm{H}_{5}
\end{gathered}
$$

where, $E_{1}$ and $E_{2}=N, P, A s, S b, B i$.

We propose to modify the Gabriel Rearrangement E-carboxymethyl and E-phenacylphalimids in a substituted 1,4-dioksiizohinoliny under the action of sodium alcoholate RONa:

$$
\begin{gathered}
\mathrm{C}_{6} \mathrm{H}_{4}(\mathrm{CO})_{2} \mathrm{ECH}_{2} \mathrm{COR} \rightarrow \\
\mathrm{C}_{6} \mathrm{H}_{4}(\mathrm{COOR})-\mathrm{COEHCH}{ }_{2} \mathrm{COR} \rightarrow \\
\mathrm{C}_{9} \mathrm{H}_{4} \mathrm{E}(\mathrm{OH})(\mathrm{OH}) \mathrm{COR}
\end{gathered}
$$

where, $\mathrm{E}=\mathrm{N}, \mathrm{P}, \mathrm{As}, \mathrm{Sb}, \mathrm{Bi}$; R = OR', $\mathrm{n}-\mathrm{C}_{6} \mathrm{H}_{4} \mathrm{R}^{\prime \prime}\left(\mathrm{R}^{\prime \prime}=\right.$ $\left.\mathrm{H}, \mathrm{CH}_{3}, \mathrm{OCH}_{3}, \mathrm{Cl}, \mathrm{Br}, \mathrm{NO}_{2}\right) ; \mathrm{R}^{\prime}=\mathrm{CH}_{3}, \mathrm{C}_{2} \mathrm{H}_{5}$.

We propose to modify the Hofmann rearrangement by replacing nitrogen atoms per atom of phosphorus, arsenic, antimony and bismuth:

$$
\begin{gathered}
\mathrm{R}-\mathrm{CO}-\mathrm{EH}_{2}+\mathrm{Br}_{2} \rightarrow[\mathrm{R}-\mathrm{E}=\mathrm{C}=\mathrm{O}] \\
+\mathrm{H}_{2} \mathrm{O} \rightarrow \mathrm{R}^{-\mathrm{EH}_{2}}
\end{gathered}
$$

where, $\mathrm{E}=\mathrm{N}, \mathrm{P}, \mathrm{As}, \mathrm{Sb}, \mathrm{Bi}$.

We propose to modify the Hofmann A.W. Martius thermal rearrangement hydrochlorides E-alkylanilines in C-alkylanilines:

$$
\begin{gathered}
\mathrm{C}_{6} \mathrm{H}_{5} \mathrm{NHR} . \mathrm{HX} \rightarrow\left[\mathrm{C}_{6} \mathrm{H}_{5} \mathrm{NH}_{2}+\mathrm{R}^{+}+\mathrm{X}^{-}\right] \rightarrow \\
\text { R- } \mathrm{C}_{6} \mathrm{H}_{4} \mathrm{NH}_{2} \cdot \mathrm{HX}
\end{gathered}
$$

where, $\mathrm{E}=\mathrm{N}, \mathrm{P}, \mathrm{As}, \mathrm{Sb}, \mathrm{Bi} ; \mathrm{X}=\mathrm{Cl}, \mathrm{Br}, \mathrm{J}$.

We propose to modify the Dimroth rearrangement by replacing nitrogen atoms per atom of phosphorus, arsenic, antimony and bismuth:
$\mathrm{R}^{\prime} \mathrm{C}_{4} \mathrm{H}_{2} \mathrm{NR}=\mathrm{EH} \rightarrow \mathrm{R}^{\prime} \mathrm{C}_{4} \mathrm{H}_{2} \mathrm{~N}_{2}$-EHR

where, $\mathrm{E}=\mathrm{N}, \mathrm{P}, \mathrm{As}, \mathrm{Sb}, \mathrm{Bi} ; \mathrm{R}=\mathrm{Alk}, \mathrm{CH}_{2} \mathrm{COOC}_{2} \mathrm{H}_{5}$; $\mathrm{R}^{\prime}=\mathrm{Alk}, \mathrm{Hal}, \mathrm{NO}_{2},\left(\mathrm{CH}_{3}\right)_{2} \mathrm{~N}, \mathrm{CONH}_{2}$.

We propose to modify the Semmler-Wolff-Schroeter rearrangement of oxi(P, As, $\mathrm{Sb}, \mathrm{Bi})$ mes $\alpha, \beta$-Unsaturated alicyclic ketones in aromatic am(arsines, stibines, bismuth)ines under the action of a mixture of the Beckmann $\left(\left(\mathrm{CH}_{3} \mathrm{CO}\right)_{2}+\right.$ $\left.\mathrm{CH}_{3} \mathrm{COOH}, \mathrm{HCl}, 100^{\circ} \mathrm{C}\right)$ :

$$
\begin{gathered}
\mathrm{RC}_{6} \mathrm{H}_{8}=\mathrm{EOH}+\left(\mathrm{CH}_{3} \mathrm{CO}\right)_{2}+\mathrm{CH}_{3} \mathrm{COOH} \rightarrow \\
{\left[\mathrm{RC}_{6} \mathrm{H}_{8}=\mathrm{EOCOCH}_{3} \rightarrow\right.} \\
\left.\mathrm{RC}_{6} \mathrm{H}_{8}=\mathrm{E}^{+} \mathrm{HOCOCH}_{3}\right] \rightarrow \\
\mathrm{RC}_{6} \mathrm{H}_{5}-\mathrm{EH}_{2}
\end{gathered}
$$

where, $\mathrm{E}=\mathrm{N}, \mathrm{P}, \mathrm{As}, \mathrm{Sb}, \mathrm{Bi} ; \mathrm{R}=\mathrm{H}$, Alk, pyridyl.

We propose to modify the Sus photochemical rearrangement of o-hinondi $(\mathrm{P}, \mathrm{As}, \mathrm{Sb}, \mathrm{Bi})$ azides in ketenes, accompanied by a narrowing of the cycle by replacing the nitrogen atoms of phosphorus, arsenic, antimony and bismuth:

$$
\begin{gathered}
\mathrm{C}_{10} \mathrm{H}_{6}(=\mathrm{O})=\mathrm{E}^{+}=\mathrm{E}^{-} \leftrightarrow \mathrm{C}_{10} \mathrm{H}_{6}\left(\mathrm{O}^{-}\right)-\mathrm{E}^{+}=\mathrm{E}+\mathrm{h} v \rightarrow \\
{\left[\mathrm{C}_{10} \mathrm{H}_{6}^{+}\left(\mathrm{O}^{-}\right) \leftrightarrow \mathrm{C}_{10} \mathrm{H}_{6}^{+}(=\mathrm{O}):\right] \rightarrow} \\
\mathrm{C}_{9} \mathrm{H}_{6}=\mathrm{C}=\mathrm{O}+\mathrm{H}_{2} \mathrm{O} \rightarrow \mathrm{C}_{9} \mathrm{H}_{6}-\mathrm{COOH}
\end{gathered}
$$

where, $\mathrm{E}=\mathrm{N}, \mathrm{P}, \mathrm{As}, \mathrm{Sb}, \mathrm{Bi}$.

We propose to modify the The thermal Claisen rearrangement by replacing nitrogen atoms phosphorus, arsenic, antimony and bismuth. Like the allylic ethers rearranged ene ( $\mathrm{P}, \mathrm{As}, \mathrm{Sb}, \mathrm{Bi})$ amines, and forming (P, As, $\mathrm{Sb}, \mathrm{Bi}$ ) mine (phospho, APCO, stibolyl, bismuth-Claisen rearrangement-phospho, arso, stibo, bismutho-Klaisen) :

$$
\begin{aligned}
& \mathrm{CH}_{2}=\mathrm{CH}-\mathrm{CH}_{2}-\mathrm{NR}-\mathrm{CH}=\mathrm{C}(\mathrm{R})-\mathrm{CH}_{3} \rightarrow \\
& \mathrm{CH}_{2}=\mathrm{CH}-\mathrm{CH}_{2}-\mathrm{C}(\mathrm{R})\left(\mathrm{CH}_{3}\right)-\mathrm{CH}=\mathrm{NR}
\end{aligned}
$$

where, $\mathrm{E}=\mathrm{C}, \mathrm{N}, \mathrm{S}, \mathrm{P}, \mathrm{As}, \mathrm{Sb}, \mathrm{Bi} ; \mathrm{R}=\mathrm{Alk}, \mathrm{C}_{6} \mathrm{H}_{5}$.

We propose to modify the Newman-Kwart thermal rearrangement of O-aryltiocarbamats in S-aryltiocarbanats:

$$
\operatorname{Ar}-\mathrm{O}-\mathrm{C}(=\mathrm{S})-\mathrm{ER}_{2} \rightarrow \operatorname{Ar}-\mathrm{S}-\mathrm{C}(=\mathrm{O})-\mathrm{ER}_{2}
$$

where, $\mathrm{E}=\mathrm{N}, \mathrm{P}, \mathrm{As}, \mathrm{Sb}, \mathrm{Bi}$.

We propose to modify the Orton Rearrangement E-halo-E-acylarilamins in o- and n-haloaryl (P, As, Sb, Bi) under the action of acid amides $\left(\mathrm{CH}_{3} \mathrm{COOH}\right)$ : 


$$
\begin{aligned}
& \text { R- } \mathrm{C}_{6} \mathrm{H}_{4}-\mathrm{E}(\mathrm{X}) \mathrm{COR}{ }^{\prime}+\mathrm{HX} \\
\rightarrow & {\left[2 \mathrm{R}-\mathrm{C}_{6} \mathrm{H}_{4}-\mathrm{EHCOR}^{\prime}+\mathrm{X}_{2}\right] } \\
\rightarrow & \mathrm{R}-\mathrm{C}_{6} \mathrm{H}_{3}\left(\mathrm{EHCOR}^{\prime}\right)(\mathrm{p}-\mathrm{X})+ \\
& \text { R- } \mathrm{C}_{6} \mathrm{H}_{3}\left(\mathrm{EHCOR}^{\prime}\right)(\mathrm{o}-\mathrm{X})
\end{aligned}
$$

where, $\mathrm{E}=\mathrm{N}, \mathrm{P}, \mathrm{As}, \mathrm{Sb}, \mathrm{Bi} ; \mathrm{X}=\mathrm{Cl}, \mathrm{Br}, \mathrm{J}$.

We propose to modify the Pistschimuka aromatic conversion ( $\mathrm{P}, \mathrm{As}, \mathrm{Sb}, \mathrm{Bi})$ amines azo $(\mathrm{P}, \mathrm{As}, \mathrm{Sb}, \mathrm{Bi})$ compound under the action of selenium (or sulfur) and merkuratsetamide:

$$
\begin{gathered}
2 \mathrm{ArEH}_{2}+\mathrm{Se}+\left(\mathrm{CH}_{3} \mathrm{CONH}\right)_{2} \mathrm{Hg} \rightarrow \\
\mathrm{Ar}-\mathrm{E}=\mathrm{E}-\mathrm{Ar}
\end{gathered}
$$

where, $\mathrm{E}=\mathrm{N}, \mathrm{P}, \mathrm{As}, \mathrm{Sb}, \mathrm{Bi} ; \mathrm{Ar}=\mathrm{C}_{6} \mathrm{H}_{4} \mathrm{R}, \mathrm{R}=\mathrm{H}, \mathrm{CH}_{3}$, $\mathrm{OCH}_{3}, \mathrm{NO}_{2}, \mathrm{Cl}, \mathrm{N}\left(\mathrm{CH}_{3}\right)_{2}$.

We propose to expand and modify Robev regrouping fenylhydraz (phospho, arso, stibo, bismutho) new aromatic aldehydes in new $\mathrm{N}$-phenylamid (phosph, ars, stibo, bismuth)ins reacting with alkalimetal amides $\left(\mathrm{KNH}_{2}, \mathrm{NaNH}_{2}\right)$ in the presence of oxidants when heated:

$$
\begin{aligned}
& \mathrm{ArCH}=\mathrm{E}-\mathrm{EHC}_{6} \mathrm{H}_{5}+\mathrm{NaEH}_{2}+\mathrm{O}_{2} \rightarrow \\
& {\left[\mathrm{ArCH}^{*}=\mathrm{EH}+* \mathrm{EHC}_{6} \mathrm{H}_{5} \rightarrow\right.}
\end{aligned}
$$

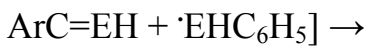

$$
\begin{aligned}
& \operatorname{ArC}(=\mathrm{EH})-\mathrm{EHC}_{6} \mathrm{H}_{5}
\end{aligned}
$$

where, E = N, P, As, Sb, Bi.

We propose to modify the Smiles isomerization of substituted aromatics $\mathrm{P}, \mathrm{As}, \mathrm{Sb}, \mathrm{Bi}$ (diarilarsenids, -phosphinoxids, -bismutons, aryl esters, P, As, Sb, Biand aromatic carboxylic acids) under the action of alkalis resulting intramolecular nucleophilic substitution:

$$
\begin{gathered}
\text { R-C } \mathrm{C}_{6} \mathrm{H}_{3}(\mathrm{ZH})-\mathrm{Y}-\mathrm{C}_{6} \mathrm{H}_{4}-\mathrm{R}^{\prime} \rightarrow \\
{\left[\mathrm{R}-\mathrm{C}_{6} \mathrm{H}_{3}\left(\mathrm{Z}^{-}\right)-\mathrm{Y}-\mathrm{C}_{6} \mathrm{H}_{4}-\mathrm{R}^{\prime} \rightarrow\right.} \\
\left.\mathrm{R}-\mathrm{C}_{6} \mathrm{H}_{3}\left(\mathrm{Y}^{-}\right)-\mathrm{Z}-\mathrm{C}_{6} \mathrm{H}_{4}-\mathrm{R}^{\prime}\right] \rightarrow \\
\text { R- } \mathrm{C}_{6} \mathrm{H}_{3}(\mathrm{YH})-\mathrm{Z}-\mathrm{C}_{6} \mathrm{H}_{4}-\mathrm{R}^{\prime}
\end{gathered}
$$

where, $\mathrm{R}=\mathrm{H}, \mathrm{CH}_{3}$, Hal; R' $=\mathrm{H}, \mathrm{NO}, \mathrm{NO}_{2}$, Hal; $\mathrm{Y}=\mathrm{S}$, $\mathrm{SO}, \mathrm{SO}_{2}, \mathrm{SO}_{2} \mathrm{O}, \mathrm{O}, \mathrm{COO} ; \mathrm{ZH}=\mathrm{OH}, \mathrm{SH}, \mathrm{NHOH}, \mathrm{NH}_{2}$, $\mathrm{AsO}_{2} \mathrm{H}, \mathrm{COSbHR}, \mathrm{BiHR} ", \mathrm{AsHR} "$ (where, R" = Alk, Ar).

We propose to modify the Rearranging Sawdey by replacing nitrogen atoms per atom of phosphorus, arsenic, antimony and bismuth by action of bases
$(\mathrm{KOH})$ :

$$
\begin{gathered}
\operatorname{ArNHN}=\mathrm{C}_{3} \mathrm{EO}(=\mathrm{O})-\mathrm{C}_{6} \mathrm{H}_{5} \rightarrow \\
\mathrm{HOOC}-\mathrm{C}_{3} \mathrm{E}_{3}(\mathrm{Ar})-\mathrm{C}_{6} \mathrm{H}_{5}
\end{gathered}
$$

where, $\mathrm{E}=\mathrm{N}, \mathrm{P}, \mathrm{As}, \mathrm{Sb}, \mathrm{Bi}$; $\mathrm{Ar}=\mathrm{C}_{6} \mathrm{H}_{5}, \mathrm{C}_{6} \mathrm{H}_{4} \mathrm{CH}_{3}$, $\mathrm{C}_{6} \mathrm{H}_{4} \mathrm{OCH}_{3}, \mathrm{C}_{6} \mathrm{H}_{4} \mathrm{Br}$.

We propose to modify the Sommelet rearrangement by replacing nitrogen atoms per atom of phosphorus, arsenic, antimony and bismuth:

$$
\begin{gathered}
\mathrm{C}_{6} \mathrm{H}_{5}-\mathrm{CH}(\mathrm{R})-\mathrm{E}^{+} \mathrm{R}_{2}{ }^{\prime}-\mathrm{CH}_{2} \mathrm{R}^{\prime \prime} \rightarrow \\
{\left[\mathrm{CHR}=\mathrm{C}_{6} \mathrm{H}_{4}-\mathrm{CH}\left(\mathrm{R}^{\prime}\right)-\mathrm{ER}_{3}{ }^{\prime}\right] \rightarrow} \\
\mathrm{CH}_{2} \mathrm{R}-\mathrm{C}_{6} \mathrm{H}_{4}-\mathrm{CH}\left(\mathrm{R}^{\prime \prime}\right)-\mathrm{ER}_{2}{ }^{\prime}
\end{gathered}
$$

where, $\mathrm{E}=\mathrm{N}, \mathrm{P}, \mathrm{As}, \mathrm{Sb}, \mathrm{Bi}$.

We propose to modify the Stevens intramolecular rearrangement quaternary $(\mathrm{P}, \mathrm{As}, \mathrm{Sb}, \mathrm{Bi})$ ammonium salts in the tertiary (phosphins, arsines, stibines, bismuthines) amines under the influence of bases:

$$
\begin{gathered}
\mathrm{RCH}_{2}-\mathrm{E}^{+}\left(\mathrm{CH}_{2} \mathrm{Ar}\right)\left(\mathrm{CH}_{3}\right)_{2} \mathrm{Br}^{-} \rightarrow \\
\mathrm{RCH}\left(\mathrm{CH}_{2} \mathrm{Ar}\right)-\mathrm{E}\left(\mathrm{CH}_{3}\right)_{2}
\end{gathered}
$$

$\mathrm{RCO}-\mathrm{E}-\mathrm{E}^{+} \mathrm{R}^{\prime}\left(\mathrm{CH}_{3}\right)_{2} \rightarrow \mathrm{RE}=\mathrm{C}=\mathrm{O}+\left(\mathrm{CH}_{3}\right)_{2} \mathrm{NR}^{\prime}(72)$ where, $\mathrm{E}=\mathrm{N}, \mathrm{P}, \mathrm{As}, \mathrm{Sb}, \mathrm{Bi} ; \mathrm{R}=\mathrm{C}_{6} \mathrm{H}_{5} \mathrm{CH}_{3}$, $\mathrm{CH}_{2}-\mathrm{CH}=\mathrm{CH}_{2}$.

We propose to modify the Tiemann Rearranging amidoximes carboxylic acid in derivatives of urea by reaction with benzenesulfonyl chloride $\left(\mathrm{C}_{6} \mathrm{H}_{5} \mathrm{SO}_{2} \mathrm{Cl}\right)$ and subsequent hydrolysis:

$$
\begin{gathered}
\mathrm{R}^{\prime} \mathrm{R} " \mathrm{E}_{1}-\mathrm{CR}=\mathrm{E}_{2} \mathrm{OH}+\mathrm{C}_{6} \mathrm{H}_{5} \mathrm{SO}_{2} \mathrm{Cl} \rightarrow \\
\mathrm{R}^{\prime} \mathrm{R} " \mathrm{E}_{1}-\mathrm{CR}=\mathrm{E}_{2}-\mathrm{OSO}_{2} \mathrm{C}_{6} \mathrm{H}_{5} \rightarrow \\
\mathrm{R}^{\prime} \mathrm{R} " \mathrm{E}_{1}-\mathrm{CO}-\mathrm{E}_{2} \mathrm{HR}
\end{gathered}
$$

where, $\mathrm{E}_{1}=\mathrm{E}_{2}=\mathrm{N}, \mathrm{P}, \mathrm{As}, \mathrm{Sb}, \mathrm{Bi} ; \mathrm{R}=\mathrm{C}_{6} \mathrm{H}_{5}$, $\mathrm{CH}_{2} \mathrm{C}_{6} \mathrm{H}_{5}$.

We propose to modify the Fischer-Hepp Rearrangement E-nitrozo aromatic ( $\mathrm{P}, \mathrm{As}, \mathrm{Sb}, \mathrm{Bi})$ amine in n-nitrozo ( $\mathrm{P}, \mathrm{As}, \mathrm{Sb}, \mathrm{Bi})$ anilines with acids:

$$
\begin{gathered}
\mathrm{R}-\mathrm{C}_{6} \mathrm{H}_{4}-\mathrm{E}\left(\mathrm{R}^{\prime}\right)-\mathrm{NO} \rightarrow\left[\mathrm{R}_{-}-\mathrm{C}_{6} \mathrm{H}_{4}-\mathrm{E}+\mathrm{H}\left(\mathrm{R}^{\prime}\right)-\mathrm{NO} \leftrightarrow\right. \\
\left.\mathrm{R}-\mathrm{C}_{6} \mathrm{H}_{4}-\mathrm{EHR}+\mathrm{NO}\right] \rightarrow \mathrm{R}^{\prime} \mathrm{C}_{6} \mathrm{H}_{3}\left(\mathrm{EHR} \mathrm{EH}^{\prime}\right)(\mathrm{NO})
\end{gathered}
$$
where, $\mathrm{E}=\mathrm{N}, \mathrm{P}, \mathrm{As}, \mathrm{Sb}, \mathrm{Bi}$; R = Alk, Hal, $\mathrm{COOH}$; R' $=$ Alk, Ar.

We propose to modify the Chapman Thermal isomerization of aromatic iminoether in $\mathrm{E}$, E-diarilamids aromatic carboxylic acids:

$$
\text { Ar-C(OAr")=NE-Ar' } \rightarrow \text { Ar-CO-E(Ar")-Ar' }
$$


where, $\mathrm{E}=\mathrm{N}, \mathrm{P}, \mathrm{As}, \mathrm{Sb}, \mathrm{Bi}$; $\mathrm{Ar}, \mathrm{Ar}^{\prime}=\mathrm{C}_{6} \mathrm{H}_{5}, \mathrm{C}_{6} \mathrm{H}_{4} \mathrm{Cl}$, $\mathrm{C}_{6} \mathrm{H}_{4} \mathrm{NO}_{2}, \mathrm{C}_{6} \mathrm{H}_{4} \mathrm{OCH}_{3}, \mathrm{C}_{6} \mathrm{H}_{4} \mathrm{CH}_{3} ; \mathrm{Ar}^{\prime \prime}=\mathrm{C}_{6} \mathrm{H}_{4} \mathrm{R}$ (where, $\mathrm{R}=\mathrm{H}, \mathrm{F}, \mathrm{Cl}, \mathrm{Br}, \mathrm{NO}_{2}$, Alk), naphtyl.

We propose to modify the Chattaway Rearrangement of E, E-diatsilanilinov in E-acyilaminoarylketons in the presence of $\mathrm{ZnCl}_{2}$ :

$$
\begin{gathered}
\mathrm{R}-\mathrm{C}_{6} \mathrm{H}_{4}-\mathrm{E}(\mathrm{COR})_{2} \rightarrow \\
\mathrm{R}-\mathrm{C}_{6} \mathrm{H}_{3}(4-\mathrm{COR})(\mathrm{EHCOR})
\end{gathered}
$$

where, $\mathrm{E}=\mathrm{N}, \mathrm{P}, \mathrm{As}, \mathrm{Sb}, \mathrm{Bi}$.

We propose to modify the Schonberg Thermal rearrangement diaryltioncarbonats in diaryltiolcarbonats by replacement of the sulfur atom to atoms of selenium and tellurium:

$$
\begin{gathered}
\operatorname{ArO}-\mathrm{C}(=\mathrm{E})-\mathrm{OAr} \rightarrow \\
{[\mathrm{Ar} \ldots \mathrm{E} \ldots \mathrm{E} \ldots \mathrm{C}-\mathrm{OAr}] \rightarrow \mathrm{Ar}-\mathrm{E}-\mathrm{C}(=\mathrm{O})-\mathrm{OAr}}
\end{gathered}
$$

where, $\mathrm{E}=\mathrm{S}$, Se, Te; $\mathrm{R}=\mathrm{Alk}, \mathrm{C}_{6} \mathrm{H}_{5} ; \mathrm{R}^{\prime}=\mathrm{H}, \mathrm{Cl}, \mathrm{Br}$, $\mathrm{CH}_{3}$.

We propose to modify the Stieglitz Rearranging triarylmethylhydroxil ( $\mathrm{P}, \mathrm{As}, \mathrm{Sb}, \mathrm{Bi})$ amines in en $(\mathrm{P}$, $\mathrm{As}, \mathrm{Sb}, \mathrm{Bi}$ ) iles under the $\mathrm{PCl}_{5}$ :

$$
\begin{gathered}
\mathrm{ArAr}_{2}{ }^{\prime} \mathrm{C}-\mathrm{EHOH} \rightarrow \\
\mathrm{Ar}_{2}{ }^{\prime} \mathrm{C}=\mathrm{E}-\mathrm{Ar}+\mathrm{ArAr}{ }^{\prime} \mathrm{C}=\mathrm{E}-\mathrm{Ar}^{\prime}
\end{gathered}
$$

where, $\mathrm{E}=\mathrm{N}, \mathrm{P}, \mathrm{As}, \mathrm{Sb}, \mathrm{Bi}$.

\section{Conclusions}

The authors' proposed new method of modifying Bamberger, Barton, Beckmann, Wallach, Gabriel, Hofmann, Hofmann A.W.-Martius, Dimroth, Semmler-Wolff-Schroeter, Sus, Claisen, Newman-Kwart, Orton, Pistschimuka, Robev, Smiles, Sawdey, Sommelet, Stevens, Tiemann, Fischer-Hepp, Chapman, Chattaway, Schonberg, Stieglitz Rearrangements opens a new opportunity to obtain new previously unknown organic compounds of arsenic, antimony and bismuth. Halides of arsenic, antimony and bismuth are convenient starting materials for the formation of C-P, C-As, C-Sb, $\mathrm{C}$-Bi-bonds in the preparation of various organometallic compounds which are more widely used as catalysts, pharmaceuticals. We have proposed a new mechanism for possible Bamberger, Barton,
Beckmann, Wallach, Gabriel, Hofmann, Hofmann A.W.-Martius, Dimroth, Semmler-Wolff-Schroeter, Sus, Claisen, Newman-Kwart, Orton, Pistschimuka, Robev, Smiles, Sawdey, Sommelet, Stevens, Tiemann, Fischer-Hepp, Chapman, Chattaway, Schonberg, Stieglitz Rearrangements.

\section{Acknowledgments}

The authors would like to thank Ruben M. Savizky (Columbia University, New York), Peter C/Burns (Notre Dame, Indiana) and Chistopher L. Cahill (George Washington University) for discussion of the results.

\section{References}

[1] Hughes, E. D., and Ingold, C. K. 1952. Quart. Rev. Chem. Soc. London 6: 48.

[2] Bamberger, E., and Landsteiner, K. 1894. Ber.

[3] Hughes, E. D., and Ingold, C. K. 1952. Quart. Rev. Chem. Soc. London 6: 45.

[4] Barton, D. H. R. 1960. J. Am. Chem. Soc. 82: 2640.

[5] Barton, D. H. R. 1961. J. Am. Chem. Soc 83: 4076.

[6] Siginome, H., Sato, N., and Masamune, T. 1969. Tetrahedron Letters 35: 3353.

[7] Jones, J., Careth, L., and Marples, B. A. 1973. J. Chem. Soc. Perkin Trans. 11: 1143.

[8] Akhtar, M. 1964. "Advances in Photochemistry." Interscience 2: 263.

[9] Kan, R. O. 1966. Organic Photochemistry. New York: McGraw-Hill.

[10] Wallach, O., and Belli, L. 1880. Ber 13: 525.

[11] Bigelow, H. E. 1931. Chem. Rev 9: 139.

[12] Chi, S. H. 1967. J. Am. Chem. Soc. 89: 4975.

[13] Hendley, E. C., and Deiffey, D. 1970. J. Org. Chem. 35: 3579.

[14] Cox, R. A. 1974. J. Am. Chem. Soc. 96: 1059.

[15] Dolenko, A., and Buncel, E. 1974. Canad. J. Chem. 52: 623.

[16] Gabriel, S., and Colman, J. 1900. Ber. 980: 2630.

[17] Allen, C. F. H. 1950. Chem. Rev. 47: 284.

[18] Hill, H. M. 1965. J. Org. Chem. 30: 620.

[19] Henecka, H. $1950 . \quad$ Chemie Der Beta-Dicarbonylverbindungen. Berlin: Springer.

[20] Hofmann, A. W. 1881. Ber.14: 2725.

[21] Wallis, E. S., and Lane, J. F. 1949. Org. React. 3: 267-306.

[22] Shioiri, T. 1991. Comp. Org. Syn. 6: 800-806.

[23] Baumgarten, H., Smith, H., and Staklis, A. 1975. The 


\section{A New Approach to Modification of Rearrangements in Metallorganic Chemistry of Phosphorus, Arsenic, Antimony and Bismuth}

Journal of Organic Chemistry 40 (24): 3554-3561.

[24] Almond, M. R., Stimmel, J. B., Thompson, E. A., and Loudon, G. M. 1993. "Hofmann Rearrangement under Mildly Acidic Conditions Using [I,I-Bis (Trifluoroacetoxy)] Iodobenzene: Cyclobutylamine Hydrochloride from Cyclobutanecarboxamide.” Org. Synth. Coll. 8: 132.

[25] Keillor, J. W., and Huang, X. 2004. "Methyl Carbamate Formation via Modified Hofmann Rearrangement Reactions: Methyl N-(p-Methoxyphenyl) Carbamate." Org. Synth. Coll. 10: 549.

[26] Hofmann, A. W., and Martius, C. A. 1871. Ber. 4: 742.

[27] Hughes, E. D., and Ingold, C. K. 1952. Quart. Rev. 6: 45.

[28] Fischer, A., Topson, R. D., and Vaughan, J. 1960. J. Org. Chem. 25: 463

[29] Odata, Y., and Tokagi, K. 1970. J. Org. Chem. 35: 1642.

[30] Dimroth, O. 1909. Ann. 364: 183.

[31] Wohren, M. 1969. Z. Chem. 9: 241.

[32] Jacquier, R., Lopez, H., and Maury, G. 1973. J. Heterocyclic. Chem. 10: 755.

[33] Saddiqui, M., Shakil, S., and Stevens, M. F. G. 1974. J. Chem. Soc. Perkin Trans. 5: 609.

[34] Brown, D. J. 1968. Mechanism of Molecular Migrations. Interscience.

[35] Semmler, F. W. 1892. Ber. 25: 3352.

[36] Wolff, L. 1902. Ann 322: 351

[37] Schroeter, G. 1930. Ber 63: 1308.

[38] Davey, J., Keene, B. R. T., and Mannering, G. 1967. J. Chem. Soc. 5: 120.

[39] Royer, R. 1970. Bull. Soc. Chim. France 3: 1026.

[40] Nunn, A. J., and Rowell, F. J. 1973. J. Chem. Soc. Perkin Trans. I 22: 2697.

[41] Sus, O. 1944. Ann556: 65.

[42] Smith, P. A., and Berry, W. L. 1961. J. Org. Chem. 26: 27.

[43] Sus, O., Munder, J., and Steppan, H. 1962. Angew. Chem. 74: 985.

[44] De, M. P. 1963. Molecular Rearrangements. London: Interscience.

[45] Claisen, L. 1912. Ber. 45: 3157.

[46] Marwell, E. N., Stephenson, J. L., and Ong, J. 1965. J. Am. Chem. Soc. 87: 1265

[47] Jefferson, A., and Scheinmann, F. 1968. Quart. Rev. 22: 391.

[48] Andrews, J. T. S. 1970. Angew. Chem. 82: 779.

[49] Mortensen, J. Z., Hedegaard, B., and Zawesson, S. 1971. Tetrahedron 27: 3831

[50] Jain, A. C., and Anand, S. M. 1974. J. Chem. Soc. Perkin Trans. 3: 329.

[51] Hartke, K., and Goelz, G. 1974. Chem. Ber. 107: 566.

[52] Gilchrist, T. L., and Storr, R. C. 1972. Organic Reactions and Orbital Symmetry. Cambridge: Univer. Press.

[53] Newman, M. S. 1966. J. Org. Chem. 31: 3980.

[54] Kwart, H., and Evans, E. R. 1966. J. Org. Chem. 31: 410.
[55] Relles, H. M., and Pizzoloto, G. 1968. J. Org. Chem. 33: 2249.

[56] Orton, K. L., and Jons, W. J. 1909. J. Chem. Soc. 95: 1456.

[57] Scott, J. M. W. 1960. Can. J. Chem. 38: 2441.

[58] Scott, J. M. W., and Martin, J. G. 1966. Can. J. Chem. 44: 2901.

[59] Pistschimuka, R. S. 1940. J. Org. Chem. (USSR) 10: 305.

[60] Pistschimuka, R. S. 1959. Ukrain J. Org. Chem. 25: 99.

[61] Robev, S. 1954. Doklads Bulgar Acad. Science 7: 37.

[62] Robev, S. 1955. Doklads Acad. Science USSR 101: 277.

[63] Naumov, U. A., and Grandberg, I. I. 1966. Uspehi Chimii 35: 21 .

[64] Warren, L. A., and Smiles, S. 1930. J. Chem. Soc. 957: 1327.

[65] Bunnett, J. F., and Zahler, R. E. 1951. Chem. Rev. 49: 362

[66] Truce, W. E., and Ray, W. J. 1959. J. Am. Chem. Soc. 81: 481-484.

[67] Schneller, S. W. 1973. Int. J. Sulfur Chem. 8: 486.

[68] Sawdey, G. W. 1957. J. Am. Chem. Soc. 79: 1955.

[69] Browne, E. J., and Polya, J. B. 1962. J. Chem. Soc. 2: 5149 .

[70] Sommelet, M. 1937. C. r. 205: 56.

[71] Hauser, C. R. 1953. J. Am. Chem. Soc. 75: 2660.

[72] Pine, S.H., and Sanchez, B. L. 1969. Tetrahedron 25: 1319.

[73] Stevens, T. S. 1928. J. Chem. Soc. 3193: 1932.

[74] Wawzonek, S., and Yeakey, E. 1960. J. Am. Chem. Soc. 82: 5718.

[75] Wadsworth, W. S. 1966. J. Org. Chem. 31: 1704.

[76] Jacobson, A. E., and Parfitt, R. T. 1967. J. Org. Chem. 32: 1894.

[77] Adams, B. L., and Kovacic, P. 1974. J. Org. Chem. 39: 3090.

[78] De, M. P. 1963. Molecular Rearrangements. London: Interscience.

[79] Tiemann, F. 1891. Ber. 24: 4162.

[80] De, M. P. 1963. Molecular Rearrangements. New York: Interscience.

[81] Fischer, O., and Hepp, E. 1886. Ber. 19: 2991.

[82] Steel, G., and Williams, D. L. 1969. Chem. Comm. 1: 975

[83] Baliga, B. T. 1970. J. Org. Chem. 35: 2031.

[84] Williams, D. L., and Wilson, J. A. 1974. J. Chem. Soc. Perkin Trans. 2: 13.

[85] Chapman, A. W. 1925. J. Chem. Soc. 127: 1992.

[86] Relles, H. M. 1968. J. Org. Chem. 33: 2245.

[87] Wheeler, O. H., Roman, F., and Rosado, O. 1969. J. Org. Chem. 343: 966.

[88] Chattaway, D. F. 1904. J. Chem. Soc. 85: 386

[89] Derick, C. G., and Bornmann, J. H. 1913. J. Am. Chem. 

Arsenic, Antimony and Bismuth

Soc. 35: 1269.

[90] Dippy, J. F., and Moss, V. 1952. J. Chem. Soc. 2205.

[91] Schonberg, A., 1930. Ann. 483: 107.

[92] Krauch, C. H., Farid, C., and Schenck, G. O. 1965, Chem. Ber. 98: 3102.

[93] Kwart, H., and Evans, E. R. 1966. J. Org. Chem. 31: 410.

[94] Newman, M. S., and Karner, H. A. 1966. J. Org. Chem. 31: 3980 .

[95] Stieglitz, J., and Vosburgh, I. 1913. Ber. 46: 2151.
[96] Berg, S. S., and Petrov, V. 1952. J. Chem. Soc. 26: 3713.

[97] Newman, M. S., and Hay, P. M. 1953. J. Am. Chem. Soc. 75: 2322

[98] Saunders, W. H., and Ware, J. C. 1958. J. Am. Chem. Soc. 80: 3328.

[99] Kovacic, P., Lowery, K. M., Field, K. W. 1970. Chem. Rev. 70: 664.

[100] Mayo, P. 1963. Molecular Rearrangements. London: Interscience. 